\title{
Effects of Discontinuous Percoll Gradient Containing Alpha-linolenic Acid on Characteristics of Frozen-thawed Boar Spermatozoa
}

\author{
Doo-San Kim ${ }^{1}$, Yong Hwangbo ${ }^{1}$, Hee-Tae Cheong ${ }^{2}$ and Choon-Keun Park ${ }^{1, *}$ \\ ${ }^{1}$ College of Animal Life Sciences, Kangwon National University, Chuncheon 24341, Korea \\ ${ }^{2}$ College of Veterinary Medicine, Kangwon National University, Chuncheon 24341, Korea
}

Received December 17, 2019

Revised January 31, 2020

Accepted February 6, 2020

\section{*Correspondence}

Choon-Keun Park

E-mail: parkck@kangwon.ac.kr

ORCID

https://orcid.org/0000-0003-2786-8814

\begin{abstract}
This present study was conducted to investigate protective effect of discontinuous Percoll gradient containing alpha-linolenic acid (ALA) before freezing process on viability, acrosome damage, mitochondrial activity, and oxidative stress of frozen-thawed boar spermatozoa. The separation of spermatozoa by discontinuous Percoll gradient was performed by different concentration of Percoll solution (45/90\%) containing ALA combined with bovine serum albumin (BSA), and collected sperm in each Percoll layer was cryopreserved. To evaluate viability, acrosome damage, mitochondrial activity, and reactive oxygen species (ROS) level of frozen-thawed sperm, flow cytometry was used. Morphological abnormalities were observed under light microscope. In results, viability of sperm from $90 \%$ Percoll layer was higher than control and $45 \%$ Percoll group ( $p<0.05$ ). Separated sperm in 90\% Percoll layer had lower acrosome damage and morphological abnormalities than control as well as viability, whereas $45 \%$ Percoll group was higher $(p<0.05)$. Similar with acrosome damage and abnormalities, mitochondrial activity was slightly enhanced and the population of live sperm with high ROS level was decreased by $90 \%$ Percoll separation, however, there was no significant difference. Supplementation of $3 \mathrm{ng} / \mathrm{mL}$ ALA into Percoll solution increased sperm viability and decreased population of live sperm with high ROS compared to control $(p<0.05)$. In conclusion, discontinuous Percoll gradient before freezing process could improve efficiency of cryopreservation of boar sperm through selection of sperm with high freezing resistance, and supplement of ALA during Percoll gradient might contribute suppression of ROS generation via stabilizing of plasma membrane during cryopreservation.
\end{abstract}

Keywords: alpha-linolenic acid, boar spermatozoa, cryopreservation, discontinuous percoll gradient

\section{INTRODUCTION}

Cryopreservation, which is one of assisted reproductive technologies, is developed to improve limited conservation period of liquid preservation of mammalian spermatozoa
(Yoshida, 2000), and it facilitates preservation of sperm from superior individuals, prevention of contamination by virus, and selective production of individuals. Despite advantages of cryopreservation, efficiency of this technique is still low because spermatozoa are exposed to damages 
on membrane function by generation of reactive oxygen species (ROS), cold shock, and formation of ice crystal during freezing and thawing process (White, 1993; Agarwal et al., 2014; Baishya et al., 2014). To reduce these damages, a variety of substrates such as antioxidants, amino acids, and cholesterol were supplied into freezing extender (Kaeoket et al., 2010; Mocé et al., 2010; Reddy et al., 2010).

Alpha-linolenic acid (ALA) is one of essential fatty acids and is classified as omega-3 fatty acid. Omega-3 fatty acids are an important indicator for fertility of spermatozoa. Safarinejad et al. (2010) reported that concentration of omega-3 fatty acids including ALA, eicosapentaenoic acid (EPA), and docosahexaenoic acid (DHA) in spermatozoa from fertile man were higher than sperm from infertile man. These omega- 3 fatty acids are found in components of feeds for domestic animals such as fish oil and sunflower oil. Because plasma membrane contains numerous polyunsaturated fatty acids (PUFAs), dietary intake of fatty acids could influence to sperm characteristics. Supplementation of sunflower and tuna oil into feed enhanced plasma membrane integrity and progressive motility of frozen-thawed sperm in bull and boar (Rooke et al., 2001; Adeel et al., 2009). These results demonstrated that additional PUFAs, especially omega-3 fatty acids, could enhanced freezing resistance of spermatozoa during cryopreservation.

Generally, mammalian semen is consisted of spermatozoa with varying degrees of morphological and functional integrity. The population of sperm with low quality have low motility, membrane integrity, and fertility. In order to enhance sperm quality, a variety of separation methods such as swim up and discontinuous Percoll gradient (DPG) were used (Jayaraman et al., 2012). Percoll is a useful tool for separation of cells, organelles, and virus, and it consists of colloidal silica beads coated with polyvinylpyrrolidone (PVP). DPG has been used for selection of subpopulation of sperm in mammalians including pigs. Parrish et al. (1995) reported that Percoll-separated bull sperm had higher motility than unseparated sperm. This technique is used for prevention of sperm contamination from virus or bacteria. Therefore, we expected that high membrane integrity of isolated spermatozoa by DPG could enhance freezing resistance during cryopreservation.

Our previous study showed that supplementation of ALA into freezing extender reduced membrane damages and ROS level of frozen-thawed boar sperm during cryo- preservation. Based on these findings, we hypothesized that addition of fatty acid into Percoll solution could enhance efficiency of cryopreservation for boar sperm. Therefore, the present study was conducted to investigate protective effect of sperm sorting by DPG containing ALA on freezing resistance of boar sperm.

\section{MATERIALS AND METHODS}

\section{Sperm samples}

All procedures that involved the use of animals were approved by the Kangwon National University Institu-tional Animal Care and Use Committee (KIACUC-09-0139). All of boar semen were purchased from Gumbo (Wonju, Korea), and the semen was collected by gloved-hand method once a week and transported to laboratory. In present study, semen from different 4 boars were used for each experiments. The fresh sperm with more than $75 \%$ motility and $80 \%$ viability, and less than $30 \%$ acrosome damage and morphological abnormalities were used.

\section{Preparation of ALA combined with bovine serum albumin (BSA)}

For supplementation of ALA into Percoll solution, ALA was mixed with BSA. Briefly, ALA and BSA were diluted to concentration at $1 \mathrm{mg} / \mathrm{mL}$ and $0.14 \%$ (v/v) using PBS without $\mathrm{Ca}^{2+}$ and $\mathrm{Mg}^{2+}$, respectively. ALA and BSA solution were mixed in different ratio (ALA : BSA $=3: 997$ and 5 : 995) and were rotated at room temperature for $1 \mathrm{~h}$. Then, $1 \mu \mathrm{L}$ of each aliquot were supplied to $1 \mathrm{~mL}$ of Percoll solution (final concentration: 3 and $5 \mathrm{ng} / \mathrm{mL}$ ALA).

\section{Sperm separation by DPG}

To perform sperm separation by two layered Percoll (45 and $90 \%, v / v)$, Percoll solution (GE healthcare UK Ltd, Buckinghamshire, England) was mixed with $10 \times$ DPBS that did not contain $\mathrm{Ca}^{2+}$ and $\mathrm{Mg}^{2+}$. Then, $2 \mathrm{~mL}$ of $45 \%$ Percoll solution was carefully layered over on $2 \mathrm{~mL}$ of 90\% Percoll solution. The sperm sample was centrifuged at 1,500 RPM for $5 \mathrm{~min}$ and supernatant was removed. The pellet was resuspended using $0.5 \mathrm{~mL}$ of Modena $\mathrm{B}$ solution and slowly dropped on $45 \%$ Percoll layer. After centrifugation at $740 \times g$ for $30 \mathrm{~min}$, sperm in $45 \%$ and 90\% Percoll layer were collected and washed twice using Modena B. The spermatozoa from different Percoll layer were immediately used for cryopreservation. 


\section{Freezing and thawing of boar spermatozoa}

As a first freezing extender, lactose egg yolk (LEY; $11 \%$ $[\mathrm{w} / \mathrm{v}$ ] lactose and $20 \%$ [v/v] egg yolk in distilled water) was used. The boar sperm was diluted at $1.5 \times 10^{9}$ spermato$\mathrm{zoa} / \mathrm{mL}$ using $1 \mathrm{~mL}$ of LEY and it was cooled down to $4^{\circ} \mathrm{C}$ for $2 \mathrm{~h}$. Then, $0.5 \mathrm{~mL}$ of LEY supplemented with 9\% Glycerol and 1.5\% Orvus Es Paste (OEP; Nova Chem, USA) was added to cooled sperm samples, and they were injected into $0.25 \mathrm{~mL}$ straws. Filled straws were placed on $10 \mathrm{~cm}$ above liquid nitrogen (LN2) for $10 \mathrm{~min}$, then, pre-freezing samples were immersed and preserved in LN2. To analyze sperm characteristics, cryopreserved samples were thawed at $37^{\circ} \mathrm{C}$ for $45 \mathrm{sec}$.

\section{Flow cytometry}

Flow cytometry was used to measure viability, acrosome damage, mitochondrial activity, and ROS level in frozenthawed boar sperm. The data from flow cytometry were obtained from total 10,000 of spermatozoa using FACs Calibur, and were analyzed by CELLQuest version 6.0. The calculation of each parameters was present in Fig. 1.

1) Measurement of viability, acrosome damage and mitochondrial activity

To measure viability, acrosome damage, and mitochondrial activity of frozen-thawed sperm, $6 \mathrm{nM}$ SYBR-14, 3 $\mu \mathrm{M}$ Lectin from Arachis hypogagea (FITC-PNA), and 2 $\mu \mathrm{M}$ Rhodamine123 were used, respectively. Samples were stained using each fluorescent dye at $38^{\circ} \mathrm{C}$ in dark room for $5 \mathrm{~min}$. Then, $2 \mu \mathrm{M}$ propidium iodide (PI) was added to stained samples and subsequently incubated in same condition for 5 min. Stained spermatozoa by only SYBR-14 was classified as viable sperm, and sperm that were positive to FITC-PNA signaling regardless PI signaling were divided as sperm with acrosome damage. Similar with acrosome damage, sperm with Rhodamine123 signaling were classified as sperm with high mitochondrial activity.

\section{2) Intracellular ROS level in live sperm}

Intracellular ROS level in live spermatozoa was measured using H2DCFDA $(0.8 \mu \mathrm{M}$, Molecular probes, USA). Thawed sperm was stained by $\mathrm{H} 2 \mathrm{DCFDA}$ at $38^{\circ} \mathrm{C}$ in dark room for $15 \mathrm{~min}$. Then, $2 \mu \mathrm{M}$ PI was added to stained samples and subsequently incubated in same condition for $5 \mathrm{~min}$. The ROS level was compared within live spermatozoa (PI negative sperm) with H2DCFDA signaling.

\section{Morphological abnormalities}

Rose Bengal staining was used to observe morphological abnormalities of frozen-thawed boar spermatozoa. The samples were smeared on slide glass and were dehydrated at room temperature. Then, samples on slide glass were stained using 3\% (v/v) Rose Bengal solution and excessive Rose Bengal solution was removed. After dehydration of stained sperm, retained Rose Bengal was washed using water and sperm samples were dehydrated for observation. The sperm with abnormal morphology were observed under a light microscopic at $\times 400$ magnification and a total of 200 spermatozoa in randomly selected five section were counted. The criteria for abnormal morphology of spermatozoa are following: head: detached, big, and small; middle piece: absence, droplet, fold, and short; tail: detached, short, two tails, and coiled.
A

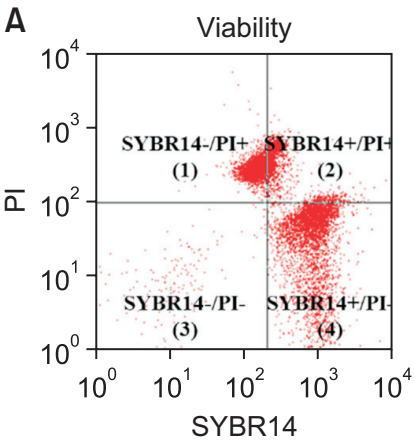

Viability $(\%)=\frac{(4)}{(1)+(2)+(3)+(4)} \times 100$

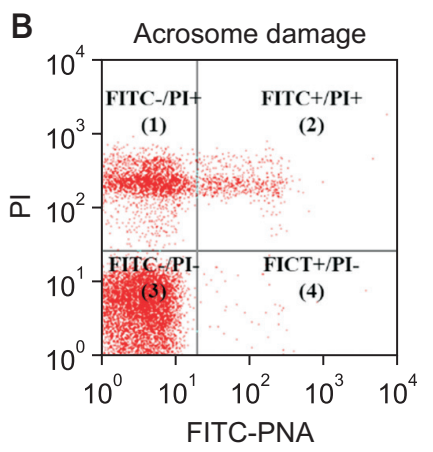

$\underset{\text { damage }(\%)}{\text { Acrosome }}=\frac{(2)+(4)}{(1)+(2)+(3)+(4)} \times 100$

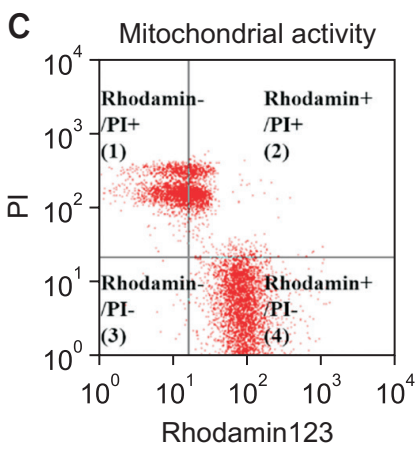

D $10^{4}$ Live sperm with high ROS

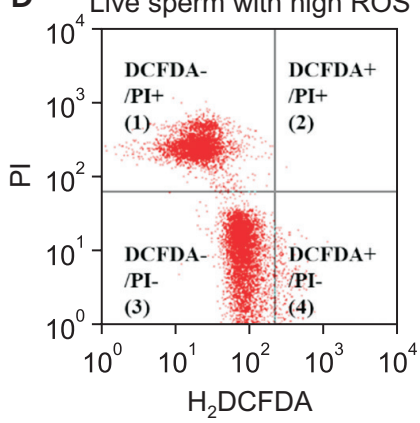

$\begin{gathered}\text { Mitochondrial } \\ \text { activity }(\%)\end{gathered}=\frac{(2)+(4)}{(1)+(2)+(3)+(4)} \times 100 \begin{gathered}\text { Live sperm with } \\ \text { high ROS }(\%)\end{gathered}=\frac{(4)}{(1)+(2)+(3)+(4)} \times 100$

Fig. 1. Dotplot images and calculation of (A) viability, (B) acrosome damage, (C) mitochondrial activity and (D) live sperm with high reactive oxygen species (ROS) in frozen-thawed boar sperm. 


\section{Statistical analysis}

All numerical data representing each parameter were analyzed using the Statistical Analysis System Software (SAS version 9.4). Data are presented means \pm SEM, and comparisons among treatment groups were conducted by Duncan's modified multiple range test using a generalized linear model (GLM) in the SAS package. A value of $p<0.05$ was considered to indicate a statistically significant difference.

\section{RESULTS}

\section{Comparison of frozen-thawed sperm characteristics from different Percoll layer}

Table 1 showed viability, acrosome damage and mitochondrial activity of frozen-thawed boar spermatozoa after DPG. The sperm in 90\% Percoll separated group had higher viability than control and $45 \%$ Percoll separated sperm ( $p<0.05)$, whereas $45 \%$ Percoll separated group did not differ compared to control group. Acrosome damage was decreased in sperm from 90\% Percoll layer and increased in sperm from $45 \%$ Percoll layer compared to control group $(p<0.05)$. Mitochondrial activity in $90 \%$ Percoll group was higher than $45 \%$ Percoll group ( $p<$ 0.05), however, both of Percoll group did not differ compared to control. Morphological abnormalities were lower in $90 \%$ Percoll group and higher in $45 \%$ Percoll group

Table 1. Effects of discontinuous Percoll gradient before cryopreservation on characteristics of frozen-thawed boar sperm

\begin{tabular}{lrrr}
\hline \multirow{2}{*}{ Parameters (\%) } & Control & \multicolumn{2}{c}{ Percoll (\%) } \\
\cline { 3 - 4 } & & \multicolumn{1}{c}{$\mathbf{4 5}$} & \multicolumn{1}{c}{90} \\
\hline Viability & $50.77 \pm 3.37^{b}$ & $48.18 \pm 2.31^{b}$ & $60.14 \pm 2.36^{a}$ \\
Acrosome damage & $13.88 \pm 0.68^{b}$ & $20.01 \pm 1.71^{a}$ & $9.64 \pm 1.31^{c}$ \\
Mitochondrial activity & $55.84 \pm 4.28^{\text {ab }}$ & $48.02 \pm 3.59^{b}$ & $62.46 \pm 3.25^{a}$ \\
\hline
\end{tabular}

${ }^{a-c}$ Mean values with different superscripts are significantly difference $(p<$ 0.05). than control as well as acrosome damage (Fig. 2; $p<0.05$ ). Similar with mitochondrial activity, population of live sperm with high ROS level was lower in sperm from $90 \%$ Percoll than 45\% Percoll (Fig. 3; $p<0.05$ ), however, both of Percoll groups were no significant difference compared to control.

\section{Effect of DPG containing ALA on frozen-thawed sperm}

Changes of sperm parameters by DPG containing ALA were showed in Table 2. Treatment of $3 \mathrm{ng} / \mathrm{mL}$ ALA into both of Percoll layer enhanced sperm viability compared to control group and $5 \mathrm{ng} / \mathrm{mL}$ ALA group $(p<0.05)$, and BSA supplementation into Percoll solution did not influenced to sperm viability. Acrosome damage in $3 \mathrm{ng} / \mathrm{mL}$ ALA group was lowest and it was significantly lower than $5 \mathrm{ng} / \mathrm{mL}$ ALA $(p<0.05)$, however, all of treatment groups were no significant difference compared to control group. Mitochondrial activity was slightly enhanced by $3 \mathrm{ng} / \mathrm{mL}$ ALA supplementation, however, there was no significant difference. The population of live sperm with high ROS

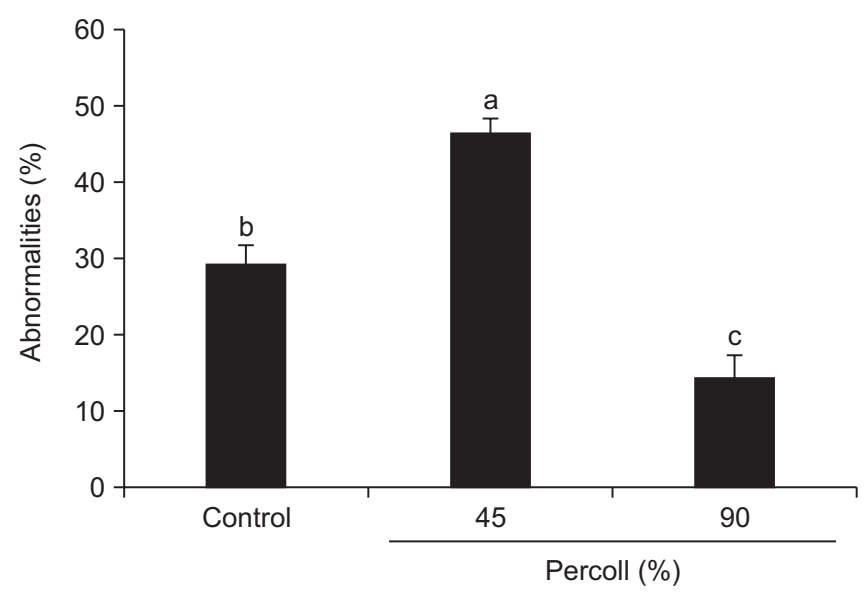

Fig. 2. Comparison of morphological abnormalities in frozenthawed boar sperm between isolated from different concentration of Percoll solution before freezing process. ${ }^{\text {a-c }}$ Superscripts indicate significant difference $(p<0.05)$.

Table 2. Effects of discontinuous Percoll gradient containing alpha-linolenic acid (ALA) before cryopreservation on characteristics of frozenthawed boar sperm

\begin{tabular}{|c|c|c|c|c|}
\hline \multirow{2}{*}{ Parameters (\%) } & \multirow{2}{*}{ Control } & \multicolumn{3}{|c|}{ ALA concentration $(\mathrm{ng} / \mathrm{mL})$} \\
\hline & & 0 & 3 & 5 \\
\hline Viability & $52.94 \pm 2.30^{b c}$ & $55.18 \pm 3.64^{\mathrm{ab}}$ & $61.25 \pm 1.89^{a}$ & $46.90 \pm 2.10^{c}$ \\
\hline Mitochondrial activity & $59.96 \pm 1.89$ & $60.98 \pm 1.52$ & $63.75 \pm 1.93$ & $58.26 \pm 2.04$ \\
\hline
\end{tabular}

${ }^{a-c}$ Mean values with different superscripts are significantly difference $(p<0.05)$. 


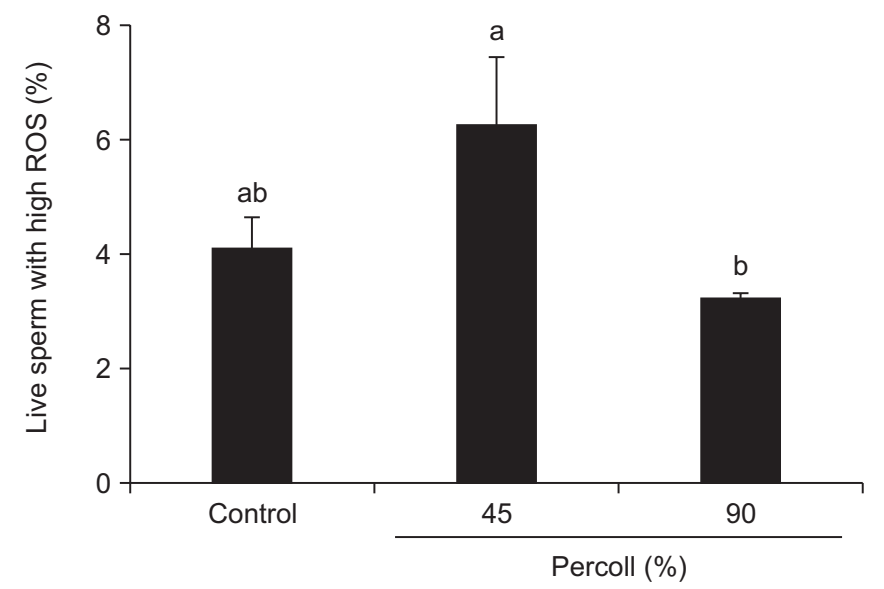

Fig. 3. Effects of discontinuous Percoll gradient before freezing process on population of live sperm with high reactive oxygen species (ROS) in frozen-thawed boar sperm. ${ }^{\text {a,b }}$ Superscripts indicate significant difference $(p<0.05)$.

was decreased in $3 \mathrm{ng} / \mathrm{mL}$ ALA treatment compared to control and $5 \mathrm{ng} / \mathrm{mL}$ ALA treatment (Fig. 4; $p<0.05$ ).

\section{DISCUSSION}

This present study was aimed to investigate changes of viability, acrosome damage, mitochondrial activity, and ROS level in frozen-thawed boar sperm that are isolated by DPG containing ALA before freezing process. Viability in sperm from 90\% Percoll layer was higher and acrosome damage was lower than unseparated and 45\% Percollseparated sperm. Supplementation of $3 \mathrm{ng} / \mathrm{mL}$ ALA into Percoll solution enhanced viability and reduced population of live sperm with high ROS level of frozen-thawed boar sperm.

During freezing and thawing process of cryopreservation, spermatozoa were exposed to damages on membrane structure such as redistribution of lipids and proteins (Parks and Graham, 1992). The membrane damages leaded to reduce membrane fluidity and permeability by releasing of fatty acids in plasma membrane (Watson, 2010), and these caused to decrease viability, motility, and induced acrosome reaction in frozen-thawed sperm. Therefore, selection of sperm with high functional integrity before cryopreservation could reduce detrimental effects of freezing and thawing process.

As one of method for sperm separation, DPG is useful tool for selection of sperm with high fertility. In present study, boar spermatozoa from $90 \%$ Percoll layer had higher plasma membrane integrity and normal morphologies,

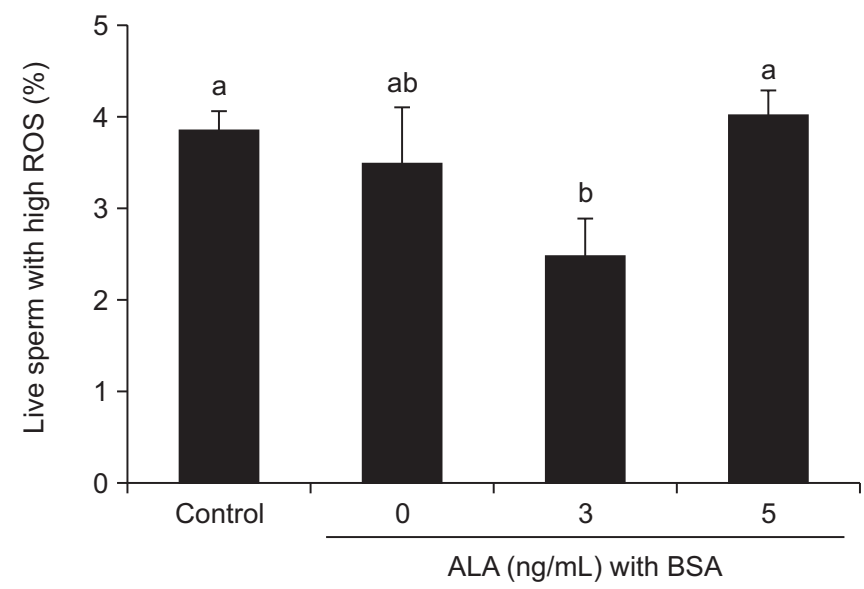

Fig. 4. Effects of discontinuous Percoll gradient containing alphalinolenic acid ( $A L A ; 0,3$ and $5 \mathrm{ng} / \mathrm{mL}$ ) combined with bovine serum albumin (BSA) before freezing process on population of live sperm with high reactive oxygen species (ROS) in frozen-thawed boar sperm. ${ }^{\mathrm{a}, \mathrm{b}}$ Superscripts indicate significant difference $(p<0.05)$.

and lower acrosome damage than both of unseparated and $45 \%$ Percoll separated sperm after cryopreservation. Matás et al. (2011) reported that DPG of boar sperm by two layer Percoll solution (45/90\%) increased population of morphological normal sperm, chromatin decondensation, and penetration into oocyte, and decreased sperm with cytoplasmic droplets, abnormal tail, and DNA fragmentation. In pigs, Percoll separation of frozen-thawed sperm enhanced total and progressive motility, penetration into oocytes, and embryonic development, whereas population of sperm with damaged acrosome membrane were reduced (Noguchi et al., 2015). These researches indicated that only sperm with intact plasma membrane are isolated by DPG and they might have higher freezing resistance.

During the sperm preservation, BSA plays roles in preventing of damage in spermatozoa. It was known to stabilize a plasma membrane of sperm by regulating of membrane fluidity (Cheng et al., 2015). Because plasma membrane contains hydrophilic outer side of phospholipid bilayer, solubility and efficiency of fatty acid supplementation are low (Graham and Foote, 1987). Thus, BSA is used as carrier protein for fatty acids. As precursor of EPA and DHA, ALA plays roles as energy resource and regulator of membrane function (Shevchenko and Simons, 2010). In our results, BSA was used as carrier proteins for ALA transport and it did not influence to sperm characteristics. However, treatment of $3 \mathrm{ng} / \mathrm{mL}$ ALA combined with BSA during DPG increased viability and decreased 
population of sperm with high ROS of frozen-thawed boar sperm. The structural and functional integrity of plasma membrane are closely associated with viability of spermatozoa. During the cryopreservation, phospholipids including ALA and arachidonic acid were released from plasma membrane, and they were caused to lose the function of membrane. Supplementation of ALA during freezing process of bull sperm enhanced motility, plasma membrane and acrosome integrity of frozen-thawed sperm (Kaka et al., 2015). Towhidi and Parks (2012) reported that PUFAs content in sperm membrane were elevated by addition of ALA into extender.

The plasma membrane of boar spermatozoa includes higher concentration of fatty acids than other species. These features of boar sperm caused to sensitive response to ROS generation and lipid peroxidation (LPO) (Tavilani et al., 2006). During cryopreservation, efflux of fatty acids from plasma membrane induced ROS generation and ROS-induced LPO, and repeated PUFAs is induced by LPO via activation of phospholipase A2 (Wathes et al., 2007). These processes lead to loss of sperm function such as motility, viability and acrosome integrity (Schiller et al., 2000; O'Connell et al., 2002; Kadirvel et al., 2009). Therefore, ALA supplementation into Percoll solution could suppress generation of ROS and ROS-induced LPO through stabilization of plasma membrane.

In our present study, boar sperm from 90\% Percoll layer had increased viability and decreased acrosome damage, and $3 \mathrm{ng} / \mathrm{mL}$ ALA in Percoll solution enhanced viability and reduced ROS level in frozen-thawed boar sperm. In conclusion, our findings showed that selected boar sperm by DPG have higher freezing resistance than unseparated spermatozoa and supplementation of ALA during DPG could reduce cryo-damages by stabilization of function in plasma membrane including fluidity.

\section{CONFLICTS OF INTEREST}

No potential conflict of interest relevant to this article was reported.

\section{ACKNOWLEDGEMENTS}

This work was supported by the National Research Foundation of Korea (NRF) grant funded by the Korea government (Ministry of Education) (2019R1A2C1004307).

\section{AUTHOR CONTRIBUTIONS}

DS Kim; Performed experiments and analyzed data. Y Hwangbo; Designed experiments and wrote the paper. HT Cheong; Supervied the research. CK Park; Designed experiments and corresponded paper.

\section{AUTHOR'S POSITION AND ORCID NO.}

DS Kim, Undergraduate, https://orcid.org/0000-0002-2528-5386

Y Hwangbo, Ph.D Student, https://orcid.org/0000-0003-3636-3551

HT Cheong, Professor, https://orcid.org/0000-0001-8225-0262

CK Park, Professor, https://orcid.org/0000-0003-2786-8814

\section{REFERENCES}

Adeel M, Ijaz A, Aleem M, Rehman H, Yousaf MS, Jabbar MA. 2009. Improvement of liquid and frozen-thawed semen quality of Nili-Ravi buffalo bulls (Bubalus bubalis) through supplementation of fat. Theriogenology 71:1220-1225.

Agarwal A, Virk G, Ong C, du Plessis SS. 2014. Effect of oxidative stress on male reproduction. World J. Mens Health 32:1-17.

Baishya SK, Biswas RK, Kadirvel G, Deka BC, Kumar S, Sinha S, Dutta DJ, Saikia GK. 2014. Effect of conventional and controlled freezing method on the post thaw characteristics of boar spermatozoa. Anim. Reprod. Sci. 149:231-237.

Cheng S, Sheng Z, Zhu JQ, Wu XF. 2015. Effects of trehalose, bovine serum albumin, and sucrose, on the integrity of the plasma membrane of Pseudosciaena crocea semen after cryopreservation. Isr. J. Aquacult. Bamid. 67.

Graham JK and Foote RH. 1987. Effect of several lipids, fatty acyl chain length, and degree of unsaturation on the motility of bull spermatozoa after cold shock and freezing. Cryobiology 24:42-52.

Jayaraman V, Upadhya D, Narayan PK, Adiga SK. 2012. Sperm processing by swim-up and density gradient is effective in elimination of sperm with DNA damage. J. Assist. Reprod. Gen. 29:557-563.

Kadirvel G, Kumar S, Kumaresan A. 2009. Lipid peroxidation, mitochondrial membrane potential and DNA integrity of spermatozoa in relation to intracellular reactive oxygen species in liquid and frozen-thawed buffalo semen. Anim. Reprod. Sci. 114:125-134.

Kaeoket K, Chanapiwat P, Tummaruk P, Techakumphu M. 2010. Supplemental effect of varying L-cysteine concentrations on the quality of cryopreserved boar semen. Asian J. Androl. 
12:760-765.

Kaka A, Wahid H, Rosnina Y, Yimer N, Khumran AM, Sarsaifi K, Behan AA, Kaka U, Ebrahimi M. 2015. $\alpha$-Linolenic acid supplementation in BioXcell ${ }^{\circledR}$ extender can improve the quality of post-cooling and frozen-thawed bovine sperm. Anim. Reprod. Sci. 153:1-7.

Matás C, Vieira L, García-Vázquez FA, Avilés-López K, LópezÚbeda R, Carvajal JA, Gadea J. 2011. Effects of centrifugation through three different discontinuous Percoll gradients on boar sperm function. Anim. Reprod. Sci. 127:62-72.

Mocé E, Blanch E, Tomás C, Graham JK. 2010. Use of cholesterol in sperm cryopreservation: present moment and perspectives to future. Reprod. Domest. Anim. 45 Suppl 2:57-66.

Noguchi M, Yoshioka K, Hikono H, Iwagami G, Suzuki C, Kikuchi K. 2015. Centrifugation on Percoll density gradient enhances motility, membrane integrity and in vitro fertilizing ability of frozen-thawed boar sperm. Zygote 23:68-75.

O'Connell M, McClure N, Lewis SE. 2002. The effects of cryopreservation on sperm morphology, motility and mitochondrial function. Hum. Reprod. 17:704-709.

Parks JE and Graham JK. 1992. Effects of cryopreservation procedures on sperm membranes. Theriogenology 38:209-222.

Parrish JJ, Krogenaes A, Susko-Parrish JL. 1995. Effect of bovine sperm separation by either swim-up or Percoll method on success of in vitro fertilization and early embryonic development. Theriogenology 44:859-869.

Reddy SSN, Jagan Mohanarao G, Atreja SK. 2010. Effects of adding taurine and trehalose to a tris-based egg yolk extender on buffalo (Bubalus bubalis) sperm quality following cryopreservation. Anim. Reprod. Sci. 199:183-190.

Rooke JA, Shao CC, Speake BK. 2001. Effects of feeding tuna oil on the lipid composition of pig spermatozoa and in vitro characteristics of semen. Reproduction 121:315-322.

Safarinejad MR, Hosseini SY, Dadkhah F, Asgari MA. 2010. Relationship of omega- 3 and omega- 6 fatty acids with semen characteristics, and anti-oxidant status of seminal plasma: a comparison between fertile and infertile men. Clin. Nutr. 29:100-105.

Schiller J, Arnhold J, Glander HJ, Arnold K. 2000. Lipid analysis of human spermatozoa and seminal plasma by MALDI-TOF mass spectrometry and NMR spectroscopy - effects of freezing and thawing. Chem. Phys. Lipids 106:145-156.

Shevchenko A and Simons K. 2010. Lipidomics: coming to grips with lipid diversity. Nat. Rev. Mol. Cell Biol. 11:593-598.

Tavilani H, Doosti M, Abdi K, Vaisiraygani A, Joshaghani HR. 2006. Decreased polyunsaturated and increased saturated fatty acid concentration in spermatozoa from asthenozoospermic males as compared with normozoospermic males. Andrologia 38:173-178.

Towhidi A and Parks JE. 2012. Effect of n-3 fatty acids and $\alpha$-tocopherol on post-thaw parameters and fatty acid composition of bovine sperm. J. Assist. Reprod. Genet. 29:10511056.

Wathes DC, Abayasekara DR, Aitken RJ. 2007. Polyunsaturated fatty acids in male and female reproduction. Biol. Reprod. 77:190-201.

Watson PF. 2000. The causes of reduced fertility with cryopreserved semen. Anim. Reprod. Sci. 60-61:481-492.

White IG. 1993. Lipids and calcium uptake of sperm in relation to cold shock and preservation: a review. Reprod. Fertil. Dev. 5:639-658.

Yoshida M. 2000. Conservation of sperms: current status and new trends. Anim. Reprod. Sci. 60-61:349-355. 